\title{
Need To Support the WHO Global Expanded Rotavirus Surveillance
}

\section{to Include Other Enteropathogens}

\author{
Beckie N Tagbo* \\ Institute of Child Health \& Department of Paediatrics, University of Nigeria Teaching \\ Hospital, Nigeria
}

*Corresponding author: Beckie N Tagbo, Institute of Child Health (Old Site),

University of Nigeria Teaching Hospital, Enugu, Enugu State, Nigeria; Email: tagbobeckie@gmail.com

\section{Editorial}

Volume 2 Issue 1

Received Date: May 03, 2018

Published Date: May 09, 2018

\section{Background}

Diarrhoea is a major cause of morbidity and mortality globally especially in low income countries where mortality is still high [1,2]. According to UNICEF, "Diarrhoea is a leading killer of children, accounting for approximately 8 per cent of all deaths among children under age 5 worldwide in 2016. This translates to over 1,300 young children dying each day, or about 480,000 children a year, despite the availability of simple effective treatment" [3]. Although the diarrhea disease burden has decreased in the past decades, the worst affected parts of the world have lagged behind in terms of decline in disease incidence. Rotavirus which is responsible for about $30-40 \%$ of diarrhea associated deaths is expected to decline significantly with accelerated rotavirus vaccine introduction in many countries of the world. This has expanded the world's attention to other pathogens important in moderate to severe diarrhea disease and death. Also receiving attention is the fact that case management of children with severe diarrhoea does not address all the issues associated with diarrhoea disease episodes, as physical and cognitive development are affected by severe episodes of diarrhea. Prevention therefore requires even more attention [4].

The recent robust Global Enteric Multicenter Study (GEMS) documented Shigella and enterotoxigenic Escherichia coli (ETEC) as important pathogens (in addition to rotavirus) associated with moderate-to-severe diarrhea (MSD) in children less than 5 years of age in low income countries of the world, especially in Sub-saharan Africa and South East Asia [3,5]. Therefore, it is needful to pay more attention to pathogens, other than rotavirus in terms of surveillance and molecular characterization, as well as vaccine development.
Keywords: Diarrhoea; Children; Rotavirus; Surveillance; Norovirus; Shigella

Abbreviations: WHO: World Health Organization; GEMS: Global Enteric Multicenter Study; GRSN: Global Rotavirus Surveillance Network; TAC: TaqMan Array Card; ETEC: Enterotoxigenic Escherichia coli; MSD: Moderate to severe diarrhea.

\section{WHO NVS Network}

The World Health Organization (WHO) in collaboration with WHO Regional Surveillance Networks (and Countries' Federal Ministries of Health) established the global rotavirus surveillance network in 2008 with the main purpose of generating local robust disease burden data regarding introduction of rotavirus vaccine as well as to monitor vaccine impact [6]. The data so generated was to serve as a strong advocacy tool for a global advocacy towards the shortening of the interval between time of vaccine licensure and time of introduction of recommended vaccines into national routine immunization programmes. This would in turn ensure that the vaccines reach the greater number of children within a short time so as to avert rotavirus diarrhea related deaths. Additionally, the surveillance data would provide a pre-vaccine introduction data against which vaccine impact could be assessed following vaccine introduction. It would also monitor trends in genotypes across the globe and this would be a valuable information not only for policy makers in their choice of vaccines but also for the industry in their effort towards improving existing vaccines as well as developing new vaccines.

The rotavirus surveillance network is part of the Global New Vaccine Surveillance Network which initially 


\section{Journal of Infectious Diseases \& Travel Medicine}

commenced with the Paediatric Bacterial Meningitis Surveillance targeting the Hib vaccine, Pneumococcal conjugate vaccine and the vaccines against Neisseria Meningitidis [7].

\section{Rotavirus Surveillance}

The Global Rotavirus Surveillance Network (GRSN) consists of WHO Regional Networks and sub-regional networks. As at 2017, the global network included 108 sentinel hospitals [8]. In the rotavirus surveillance, children less than 5 years of age with acute diarrhea admitted in participating sentinel hospitals are screened for eligibility and enrolled using the Global WHO Generic protocol and Standard Operating Procedures [9]. ELISA test is carried out at the sentinel sites on stool samples collected from enrolled patients and specimens are shipped to WHO designated Regional Reference laboratories for quality control and genotyping.

Over the years, substantial achievements have been made including the generation of robust burden of disease data in all regions, mobilization of strong advocacy moves by use of these data to country policy makers on need to introduce rotavirus vaccines, use of generated data to prepare vaccine introduction application to Gavi by Gavi-eligible countries, introduction of rotavirus vaccine into national routine immunization programme in over 90 countries of the world [10], impact monitoring of rotavirus vaccines in countries that have introduced the vaccines, as well as knowledge of prevalent rotavirus strains. Indeed, nearly $50 \%$ of the countries of the world and nearly $60 \%$ of Gavieligible countries have introduced the vaccine either nationally or sub-nationally $[10,11]$.

\section{The Rotavirus Surveillance Expansion}

Following the introduction of rotavirus vaccines in many countries, the burden of rotavirus diarrhea hospitalization has dropped by about 59\% to $71 \%$ [12] and it is expected to drop further as more countries plan to introduce the vaccine in the next few years. The incidence has dropped from 528,000 deaths in year 2000 (the pre-vaccine era) to 215,000 deaths in 2013 (post vaccine introduction era) [13]. As rotavirus burden drops, diarrhoea caused by other enteropathogens becomes more visible. Attention is therefore being drawn to other enteropathogens in addition to rotavirus. Although rotavirus is still the commonest cause of severe dehydrating diarrhea in underfive children, accounting for about 40\% [14-17] of cases, other pathogens (like Norovirus, E-Coli, Shigella, Campylobacter, Gardia lamblia, etc.) still contribute significantly to morbidity and mortality.

Initially, how to test for all these pathogens within the surveillance was a huge challenge. However, recently the WHO and her partners initiated a first phase pilot study using a new molecular technique, TaqMan Array Card (TAC) technology, to further understand the importance of other enteric pathogens in the etiology of childhood diarrhoeal diseases especially in the post rotavirus vaccine era and also to facilitate vaccine development against pathogens like norovirus, ETEC, and Shigella by providing additional genotyping information for these pathogens. In the second phase, WHO regional reference laboratories are supporting the rotavirus surveillance expansion pilot project by conducting broad testing of samples for enteropathogens using quantitative PCR (via TaqMan Array Cards) [18]. This novel molecular technology though expensive, is capable of performing up to 384 simultaneous real-time PCR (qPCR) reactions and can be used for detection of Viral, bacterial, and fungal pathogens, as well as parasites [19].

It required a minor adjustment of the case definition to capture all under five diarrhoea cases, including chronic and bloody diarrhoeas. Sites were selected based on set criteria. Sample collection and shipment uses the already existing rotavirus surveillance network platforms. TAC testing is to be done at the WHO regional reference laboratories after pooling samples from participating countries together.

\section{Need for Global Support}

This is a laudable project that requires support from governments and non-governmental organizations as well as international development agencies to ensure it succeeds. Funding challenges need to be addressed due to increases in requirements for TAC testing, training of human resources, procurement of additional logistics like storage capacity and technical support [18].

Several benefits are expected from this project. Addressing a wide range of diarrhea disease causing pathogens is a basic step towards reducing underfive major cause of morbidity and mortality. The data so generated is expected to aid vaccine development against major causes of diarrhea in addition to rotavirus, ultimately leading to a greater reduction in childhood morbidity and mortality. This is a call for all to support WHO and her partners in this laudable project that is expected to further significantly reduce rotavirus disease burden among children less than 5 years of age. 


\section{Journal of Infectious Diseases \& Travel Medicine}

\section{References}

1. Liu L, Johnson HL, Cousens S, Perin J, Scott S, et al. (2012) Global, regional, and national causes of child mortality: an updated systematic analysis for 2010 with time trends since 2000. The Lancet 379 (9832): 2151-2161

2. Tagbo BN, Mwenda JM, Armah G, Obidike EO, Okafor $\mathrm{UH}$, et al. (2014) Epidemiology of rotavirus diarrhea among younger than 5 years in Enugu, South East, Nigeria. Pediatr Infect Dis J 33(1): S19-S22.

3. (2018) UNICEF Data: Monitoring the Situation of Children and Women.

4. Margaret Mokomane, Ishmael Kasvosve, Emilia de Melo, Jeffrey M. Pernica, David M (2017) Goldfarb. The global problem of childhood diarrhoeal diseases: emerging strategies in prevention and management. Therapeutic Advances in Infectious Disease 5(1): 2943.

5. Kotloff KL, Nataro JP, Blackwelder WC, Dilruba Nasrin, Tamer H Farag, et al. (2013) Burden and aetiology of diarrhoeal disease in infants and young children in developing countries (the Global Enteric Multicenter Study, GEMS): a prospective, case-control study. Lancet 382(9888): 209-222.

6. (2018) World Health Organization. Global Rotavirus Information and Surveillance Bulletin Reporting Period. 1: 1-3.

7. World Health Organization (2018) Summary Report and Action Plan for 2012 from the Global Meeting on Surveillance for Vaccine Preventable Invasive Bacterial Diseases (VP-IBD) and Rotavirus 22-24 September 2010 Geneva, Switzerland.

8. World Health Organization (2018) Rotavirus Laboratory network. Immunization, Vaccines and Biologicals.

9. World Health Organization (2018) Generic protocols for (i) hospital-based surveillance to estimate the burden of rotavirus gastroenteritis in children and (ii) a community-based survey on utilization of health care services for gastroenteritis in children.

10. O'Ryan M (2017) Rotavirus Vaccines: a story of success with challenges ahead. F1000Research 6: 1517
11. International Vaccine Access Center (IVAC) (2018) VIEW-hub Report: Global Vaccine Introduction and Implementation 2017: 1-28

12. Burnett E, Jonesteller CL, Tate JE, Yen C, Parashar UD, et al. (2017) Global Impact of Rotavirus Vaccination on Childhood Hospitalizations and Mortality from Diarrhea. J Infect Dis 215(11): 1666-1672.

13. Tate JE, Burton AH, Boschi-Pinto C, Parashar UD (2016) Global, Regional, and National Estimates of Rotavirus Mortality in Children < 5 Years of Age, 2000-2013. Clinical Infectious Diseases 62(suppl 2): S96-S105.

14. Enane LA, Gastañaduy PA, Goldfarb DM, Jeffrey M. Pernica, Margaret Mokomane, et al. (2016) Impact of Rotavirus Vaccination on Hospitalizations and Deaths From Childhood Gastroenteritis in Botswana. Clinical Infectious Diseases: An Official Publication of the Infectious Diseases Society of America 2(2): S168S174.

15. Hegazi MA, Sayed MH, Sindi HH, Osama Elsayed Bekhit, Basem Salama El-Deek, et al. (2017) Is rotavirus still a major cause for diarrheal illness in hospitalized pediatric patients after rotavirus vaccine introduction in the Saudi national immunization program? Shiau S (Ed.), Medicine 96(15): e6574.

16. Mwenda J, Mihigo R, Tevi-Benissan C, Mutale Mumba, Deo Nshimirimana, et al. (2015) Rotavirus disease burden in Africa and the need to accelerate introduction of vaccines. African Health Monitor 19: 1-7.

17. Esparza-Aguilar M ,Gastañaduy PA, Sánchez-Uribe E, Desai R, Parashar UD, et al. (2014) Diarrhoea-related hospitalizations in children before and after implementation of monovalent rotavirus vaccination in Mexico. Bull World Health Organ 92(2): 117-25

18. World Health Organization (2018) Meeting report Global Rotavirus and Pediatric Diarrheal Surveillance Network Meeting 16th -17th November 2017 Hotel Royal Geneva, Switzerland.

19. Diaz MH, Waller JL, Napoliello RA, Islam MS, Wolff BJ, et al. (2013) Optimization of Multiple Pathogen Detection Using the TaqMan Array Card: Application for a Population-Based Study of Neonatal Infection. PLoS ONE 8(6): e66183. 